\title{
Fate of density functional theory in high-pressure solid hydrogen
}

\author{
Sam Azadi and W. M. C. Foulkes \\ Thomas Young Centre and Department of Physics, \\ Imperial College London, London SW7 2AZ, United Kingdom
}

\begin{abstract}
This paper investigates some of the successes and failures of density functional theory in the study of high-pressure solid hydrogen at low temperature. We calculate the phase diagram, metallization pressure, phonon spectrum, and proton zero-point energy using three popular exchange-correlation functionals: the local density approximation (LDA), the Perdew-Burke-Ernzerhof (PBE) generalized gradient approximation, and the semi-local Becke-Lee-Yang-Parr (BLYP) functional. We focus on the solid molecular $\mathrm{P} 63 / \mathrm{m}, \mathrm{C} 2 / \mathrm{c}, \mathrm{Cmca}-12$, and Cmca structures in the pressure range from $100<P<500 \mathrm{GPa}$ over which phases I, II and III are observed experimentally. At the static level of theory, in which proton zero-point energy is ignored, the LDA, PBE and BLYP functionals give very different structural transition and metallization pressures, with the BLYP phase diagram in better agreement with experiment. Nevertheless, all three functionals provide qualitatively the same information about the band gaps of the four structures and the phase transitions between them. Going beyond the static level, we find that the frequencies of the vibron modes observed above $3000 \mathrm{~cm}^{-1}$ depend strongly on the choice of exchange-correlation functional, although the low-frequency part of the phonon spectrum is little affected. The largest and smallest values of the proton zero-point energy, obtained using the BLYP and LDA functionals, respectively, differ by more than $10 \mathrm{meV} /$ proton. Including the proton zero-point energy calculated from the phonon spectrum within the harmonic approximation improves the agreement of the BLYP and PBE phase diagrams with experiment. Taken as a whole, our results demonstrate the inadequacy of mean-fieldlike density functional calculations of solid molecular hydrogen in phases I, II and III and emphasize the need for more sophisticated methods.
\end{abstract}

\section{INTRODUCTION}

In 1935, Wigner and Huntington ${ }^{1}$ predicted that the molecules in solid molecular hydrogen at very high pressure would dissociate to form a metallic atomic solid. Solid hydrogen is expected to transform into a metallic liquid ground state at high pressure, $\stackrel{2}{=}$ may exhibit high$\mathrm{T}_{\mathrm{c}}$ superconductivity and superfluidity, $\underline{\underline{3}} \underline{\underline{\underline{5}}}$ and plays an important role in astrophysics $\stackrel{\underline{6}}{=}$ Although it is not yet possible to reach the static pressure of more than 400 GPa normally thought necessary to dissociate the hydrogen molecules, recent experimental results obtained using diamond anvil cell techniques have been interpreted as indicating metallization and the transition of molecular hydrogen to an atomic liquid, known as phase IV, below $300 \mathrm{GPa} \stackrel{\underline{7}}{*}$ Other experimental groups disagree, observing no evidence of the optical conductivity expected of a metal over the entire range of temperatures to the highest pressures explored, and no signature of an atomic liquid $\underline{\underline{8}}$ The interpretation of experimental results is complicated by the fact that, unlike phase I $(<110 \mathrm{GPa})$, which is a molecular solid of quantum rotors on a hexagonal close packed lattice, the structures of phase II (known as the broken-symmetry phase $\mathrm{e}^{\frac{9}{}}$ ) and phase III ( $\left.>150 \mathrm{GPa}\right)$ are unknown. $\underline{\underline{10}}$ In addition, it remains unclear whether or not phase III is metallic $\stackrel{11}{=}$

Metallization is believed to occur either via the dissociation of hydrogen molecules and a structural transformation to an atomic metallic phase or via band overlap (band-gap closure) within the molecular phase. There is more evidence in favor of the latter assumption. Quantum Monte Carlo (QMC) calculations of metallic hydrogen $\frac{12}{2}$ and our knowledge of the experimental equation of state $\frac{13}{\underline{13}}$ suggest that the hydrogen molecules remain undissociated at pressures up to $620 \mathrm{GPa}$. Density functional theory (DFT) calculations using the ab initio random structure searching approach 14 predict dissociation near $500 \mathrm{GPa}, \frac{15}{\stackrel{1}{w}}$ whilst DFT calculations using evolutionary techniques $\frac{16}{6}$ predict that the formation of stable non-molecular metallic hydrogen requires pressures much higher than $600 \mathrm{GPa}, \frac{17}{1}$ These values are so large that it seems likely that metallization takes place by band-gap closure in the molecular phase well before dissociation occurs.

Experimental vibrational and scattering results are not yet sufficient to allow an unambiguous determination of the structure of phase III, leaving theorists free to speculate $\underline{18-23}$ However, the range of structures proposed is so wide and the energy differences between them so small (typically a few $\mathrm{meV} /$ proton) that it is unclear which, if any, corresponds to the global free energy minimum. Finding the most stable arrangements of atoms in solids and molecules is and will remain a very difficult task, but significant progress has been made over the past few years. Recent advances based on simulated annealing, metadynamics, random sampling, evolutionary algorithms, basin hopping, minima hopping, and data mining, are discussed in Ref. 24 .

The ab initio random structure searching method 14 has also been used to investigate the zero-temperature phase diagram of molecular solid hydrogen ${ }^{15}$ Searches excluding the effects of proton zero-point energy (ZPE) found a sequence of molecular crystal structures within the pressure range over which phase III is observed exper- 
imentally. The C2/c structure was stable up to $270 \mathrm{GPa}$; Cmca-12 was stable from $270 \mathrm{GPa}$ to $385 \mathrm{GPa}$; and Cmca was stable above $385 \mathrm{GPa}$. The consequences of including proton ZPE (see Section IV) were investigated only after the structures had been found. Ref. 15 provided many new ideas about the phase diagram of solid molecular hydrogen, but the energies of the crystal structures considered were obtained using a simple semi-local approximation to the unknown exchange-correlation (XC) functional of density functional theory (DFT), which may or may not be accurate. Moreover, since DFT substantially underestimates the fundamental band gap for essentially all materials, it also underestimates the pressure at which the band gap closes ${ }^{25}$ Finally, it is worth noting that crystal structures obtained using random searching do not always agree with structures obtained using other techniques or experiment ${ }^{26,27}$ This is not surprising, as the probability that a random search will find the ground state decreases exponentially with increasing system size 24

DFT is one of very few quantum mechanical methods capable of calculating the energies and enthalpies of all phases of interest over all relevant pressure regimes and has played an important role in studies of high-pressure hydrogen. However, serious doubts about the accuracy of the results persist. How do the inter-atomic interaction energy, the bond-stretch energy, the phase diagram, the metallization mechanism, and the phonon spectrum depend on the approximation used for the XC functional? How accurate should we expect DFT calculations of measured quantities such as infra-red (IR) and Raman spectra to be? Answering these questions is necessary to assess the reliability of the many existing DFT simulations of high-pressure solid hydrogen. $\stackrel{28,29}{2}$ On the minus side, because the enthalpy differences between rival structures are so small (a few $\mathrm{meV} /$ proton), it is very difficult to be sure that a specific structure represents the global minimum. The failures of DFT to describe water and other systems containing hydrogen at low pressure and temperature have already been documented 30 Perhaps surprisingly, the errors of DFT are already troublesome even for a single $\mathrm{H}_{2} \mathrm{O}$ molecule: it was shown recently ${ }^{31,32}$ that some common $\mathrm{XC}$ functionals provide rather poor estimates of the $\mathrm{O}-\mathrm{H}$ bond-stretch energy. Because of the limited experimental data, theoretical and computational studies have an unusually important role to play in the study of solid hydrogen, but only if they are correct.

Our purpose in this paper is to investigate the successes and failures of several widely used XC functionals in the study of high-pressure solid hydrogen. We emphasize that the failures we identify do not signal a breakdown of DFT itself; they demonstrate only the limited accuracy of certain XC functionals. We believe that identifying and investigating deficiencies such as these provides useful information to developers of new XC functionals and may bring us closer to one of the main goals of DFT, which is to design functionals capable of providing accurate total energies for as wide a variety of systems as possible.

Starting at the static level of theory, we calculate the zero-temperature phase diagram using three different functionals: the Perdew-Zunger (PZ) parameterization of the local density approximation 33 (LDA), the Perdew-Burke-Ernzerhof (PBE) version of the generalized gradient approximation ${ }^{34}$ (GGA), and the semi-local Becke-Lee-Yang-Parr (BLYP) functional 35 We focus on four specific structures with space groups $\mathrm{P} 6_{3} / \mathrm{m}, \mathrm{C} 2 / \mathrm{c}$, Cmca-12, and Cmca. According to previous static DFT calculations using the PBE functional, $\stackrel{15}{1}$ these are stable in the pressure ranges $<105 \mathrm{GPa}, 105-270 \mathrm{GPa}, 270-385$ GPa, and 385-490 GPa, respectively.

Nuclear quantum effects are generally neglected in DFT calculations because of computational cost. However, there is strong evidence to show that this neglect has a significant impact on the results for solid hydrogen, even at finite temperature 36 We investigate quantum nuclear effects at zero temperature by calculating the proton ZPE as a function of pressure within the quasiharmonic approximation. Our calculations of the phonon spectrum also enable us to investigate the effect of the choice of $\mathrm{XC}$ functional on the frequencies of the Raman and IR active modes. We demonstrate that adding the proton ZPE to the calculated enthalpy produces " $\mathrm{dy}$ namic" phase diagrams in better agreement with experiment than the static phase diagrams obtained excluding ZPE.

The paper is organized as follows. Section II describes the details of our DFT calculations. Section III investigates the effect of the choice of $\mathrm{XC}$ functional on the calculated phase diagram and metallization pressure of solid molecular hydrogen at the static level of theory, and shows how the choice of functional affects the pressures at which the phase transitions $\mathrm{I} \rightarrow \mathrm{II} \rightarrow$ III are predicted to take place. In Section IV, we go beyond the static level by investigating the effects of ZPE. Section VI concludes.

\section{COMPUTATIONAL DETAILS}

Since the energy differences between high-pressure solid molecular structures are very small, the calculations must be done with the highest possible numerical precision. Our DFT calculations were carried out within the pseudopotential and plane-wave approach using the Quantum Espresso suite of programs $\frac{37}{2}$ All calculations used norm-conserving pseudopotentials and a basis set of plane waves with a cutoff of 200 Ry. Geometry and cell optimizations employed a dense $16 \times 16 \times 16 \mathrm{k}$-point mesh. The BFGS quasi-Newton algorithm was used for cell and geometry optimization, with convergence thresholds on the total energy and forces of $0.01 \mathrm{mRy}$ and 0.1 mRy/Bohr, respectively, to guarantee convergence of the total energy to better than $1 \mathrm{meV} /$ proton and the pressure to better than $0.1 \mathrm{GPa} /$ proton.

To include the effects of $\mathrm{ZPE}$ and investigate the 
phonon spectrum, vibrational frequencies were calculated using density-functional perturbation theory as implemented in Quantum Espresso 37 The ZPE per proton at a specific cell volume $V$ was estimated within the harmonic approximation: $E_{\mathrm{ZPE}}(V)=3 \hbar \bar{\omega} / 2$, where $\bar{\omega}=\sum_{\mathbf{q}} \sum_{i=1}^{N_{\text {mode }}} \omega_{i}(\mathbf{q}) /\left(N_{\mathbf{q}} N_{\text {mode }}\right)$. Here $N_{\text {mode }}$ and $N_{\mathbf{q}}$ are the numbers of vibrational modes in the unit cell and phonon wave vectors $\mathbf{q}$, respectively, and the summation over $\mathbf{q}$ includes all $\mathbf{k}$-points on a $2 \times 2 \times 2$ grid in the Brillouin zone. McMahon $\underline{42,43}$ demonstrated that a $\mathbf{q}-$ point grid of this density is sufficient to converge ZPE differences between structures to within a few percent. The enthalpy at pressure $P$, including ZPE effects, was obtained by minimizing $H(P)=E(V)+E_{\mathrm{ZPE}}(V)+P V$ with respect to $V$ at constant $P$, where $E(V)$ is the static ground-state energy at volume $V$.

\section{STATIC RESULTS AND DISCUSSION}

This section reports the ground-state phase diagram of solid molecular hydrogen in the pressure range corresponding to phases I, II and III $(100<P<500$ $\mathrm{GPa}$ ). Three different $\mathrm{XC}$ functionals were used to calculate the enthalpies of the Cmca, C2/c, Cmca-12, and $\mathrm{P}_{3} / \mathrm{m}$ structures found by recent $\mathrm{PBE}$ calculations $\underline{15}$ to be stable (have the lowest enthalpy) at different pressures within this range. These structures are illustrated in Figs. 1, 2, 3, and 4.

Figure 5 shows the static lattice enthalpy as a function of pressure calculated using the LDA, PBE and BLYP functionals. According to our LDA calculations, the $\mathrm{P} 66_{3} / \mathrm{m}, \mathrm{C} 2 \mathrm{c}$, Cmca-12, and Cmca phases are stable in the pressure ranges $<70 \mathrm{GPa}, 70-165 \mathrm{GPa}, 165-260$ $\mathrm{GPa}$, and $>260 \mathrm{GPa}$, respectively. Our PBE phase diagram is in agreement with previous work $\stackrel{15}{=}$ indicating that the $\mathrm{P}_{3} / \mathrm{m}, \mathrm{C} 2 / \mathrm{c}$, Cmca-12, and Cmca phases are stable in the pressure ranges $<110 \mathrm{GPa}, 110-245 \mathrm{GPa}$, 245-370 GPa, and $>370 \mathrm{GPa}$, respectively. According to experiment, the phase transition from phase I to phase II happens around $110 \mathrm{GPa}$ and is characterized by a change in the low-frequency region of the Raman and IR spectra $\stackrel{44}{\underline{4}}$ It is interesting that the structural transition from $\mathrm{P} 6_{3} / \mathrm{m}$ to $\mathrm{C} 2 / \mathrm{c}$ is also observed at $110 \mathrm{GPa}$ when the PBE functional is used in the static approximation. The static PBE phase diagram does not provide any information about the transition from phase II to III, which is observed experimentally at around $150 \mathrm{GPa}$ and is accompanied by a large low-temperature discontinuity in the Raman scattering and a strong rise in the IR molecular vibrons $\underline{\underline{44}}$ The static BLYP phase diagram, by contrast, identifies the transition from phase II to III with the structural change from $\mathrm{P}_{3} / \mathrm{m}$ to $\mathrm{C} 2 / \mathrm{c}$. As Fig. 5 (c) illustrates, BLYP calculations predict that the $\mathrm{P} 6_{3} / \mathrm{m}$, C2c, Cmca-12, and Cmca phases are stable in the pressure ranges of $<160 \mathrm{GPa}, 160-370 \mathrm{GPa}, 370-430 \mathrm{GPa}$, and $>430 \mathrm{GPa}$, respectively.

Figure [6 illustrates how the DFT band gaps of the

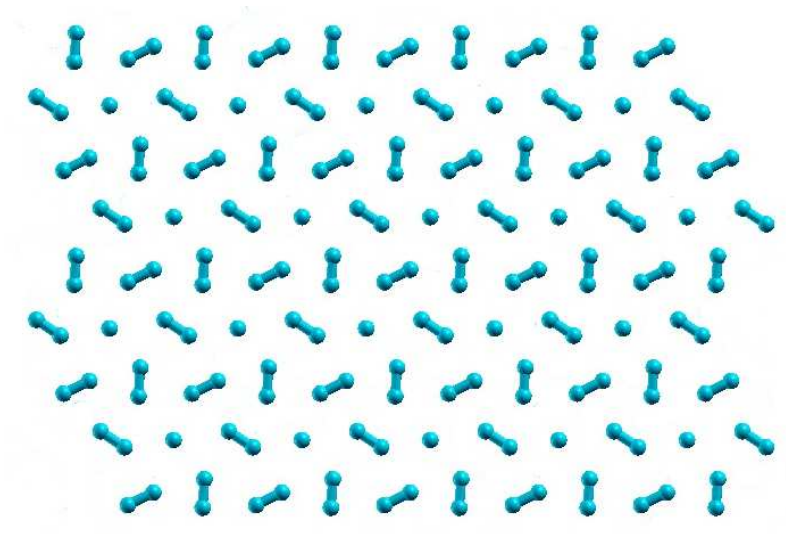

FIG. 1. (color online) A layer of the hexagonal $\mathrm{P} 66_{3} / \mathrm{m}$ structure at $300 \mathrm{GPa}$. The layers are stacked in an ABAB fashion. The primitive unit cell contains 16 atoms, which form hydrogen molecules of two types: $75 \%$ of the molecules lie flat within the plane and $25 \%$ lie perpendicular to the plane.

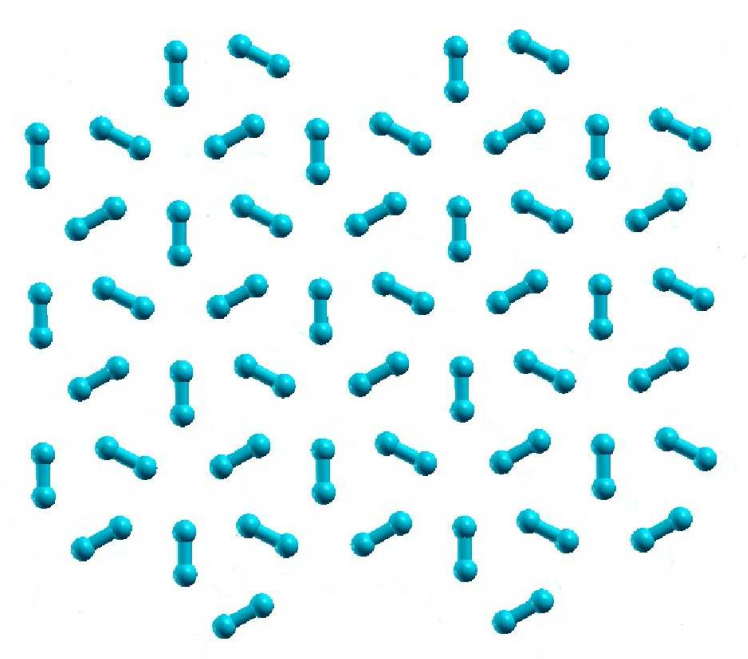

FIG. 2. (color online) A layer of the monoclinic C2/c structure at $300 \mathrm{GPa}$. The layers are arranged in an ABCDA fashion and the primitive unit cell contains 12 atoms.

Cmca- $12, \mathrm{C} 2 / \mathrm{c}$ and $\mathrm{P}_{3} / \mathrm{m}$ structures close as the pressure increases. The results obtained using different $\mathrm{XC}$ functionals differ markedly. The DFT band gap does not correspond to the measured quasi-particle band gap and is usually much smaller, so these results are not expected to agree with experiment; the true pressures at which the band gaps of these structures close are likely to be significantly higher than suggested by Fig. 6.

Exact-exchange (EXX) DFT calculations yield band gaps $1-2 \mathrm{eV}$ higher than LDA gaps $\stackrel{23}{\underline{n} \text { Although this } 1-2}$ $\mathrm{eV}$ difference was obtained by comparing LDA and EXX calculations, Pickard 15 investigated the effect of adding $1-2 \mathrm{eV}$ to the PBE band gap of the $\mathrm{C} 2 / \mathrm{c}$ phase. Increasing the PBE gap by $1 \mathrm{eV}$ or $2 \mathrm{eV}$ and extrapolating to the pressure at which the increased gap vanished gave metallization pressures of 350 and $410 \mathrm{GPa}$, respectively. Table 


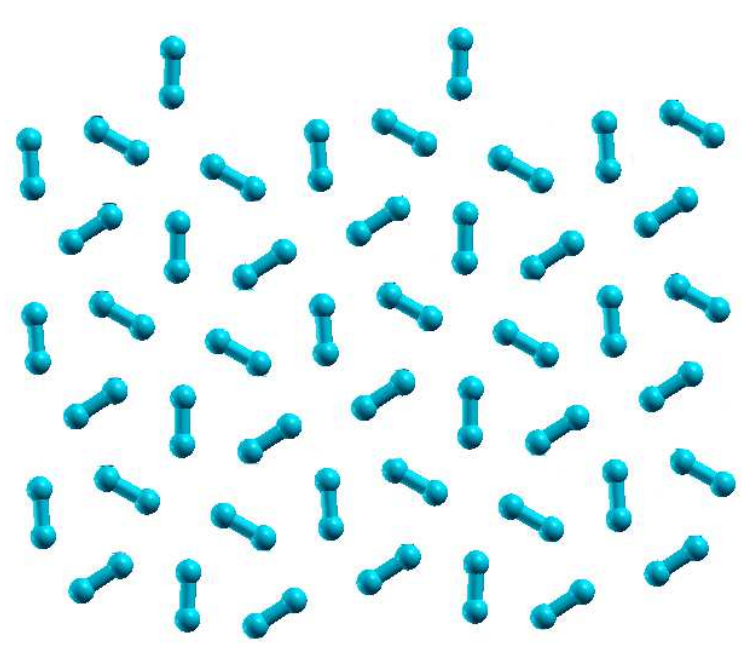

FIG. 3. (color online) A layer of the monoclinic Cmca-12 structure at $300 \mathrm{GPa}$. The layers are arranged in an ABA fashion and the primitive unit cell contains 12 atoms.

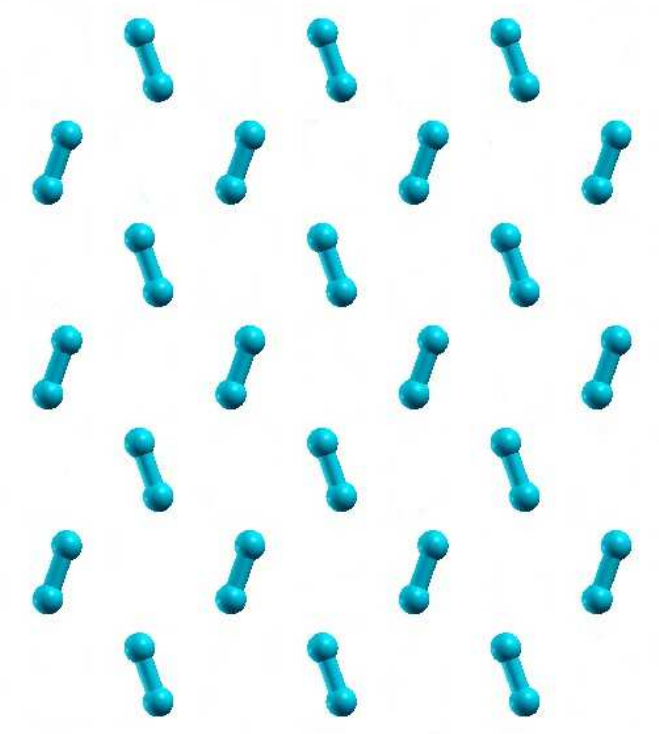

FIG. 4. (color online) A layer of the Cmca structure at 300 GPa. The layers are arranged in an ABA fashion and the primitive unit cell contains 8 atoms.

Пreports the pressures of band-gap closure obtained using the LDA, PBE and BLYP functionals with and without band-gap corrections of $1 \mathrm{eV}, 1.5 \mathrm{eV}$ and $2 \mathrm{eV}$. Use of the BLYP functional yields the highest calculated metallization pressures for all three structures, even higher than the pressures obtained by applying a $2 \mathrm{eV}$ correction to the LDA gap.

The enthalpy-pressure relationships plotted in Fig. 5 show that the LDA favors the metallic Cmca phase relative to the insulating phases, and hence that the structural transition to the Cmca phase occurs at a lower pres-
TABLE I. Calculated pressure in GPa at which the band gap of solid molecular hydrogen in the Cmca-12, C2/c and $\mathrm{P} 63 / \mathrm{m}$ structures closes. The metallization pressure is obtained by plotting the band gap as a function of pressure and extrapolating to the point at which the gap vanishes. Band gaps obtained using the LDA, PBE and BLYP XC functionals are shown. The effects of adding band-gap corrections of 1.0, 1.5 and $2.0 \mathrm{eV}$ to the LDA results are also indicated.

\begin{tabular}{|c|c|c|c|c|c|c|}
\hline \hline Space Group & LDA & LDA+1 & LDA+1.5 & LDA+2 & PBE & BLYP \\
\hline Cmca-12 & 160 & 205 & 228 & 248 & 230 & 320 \\
C2 $/ \mathrm{c}$ & 230 & 283 & 313 & 343 & 298 & 420 \\
$\mathrm{P} 63 / \mathrm{m}$ & 240 & 305 & 336 & 366 & 325 & 480 \\
\hline \hline
\end{tabular}

sure within the LDA. Furthermore, Fig. 6] shows that the band gaps of the insulating phases close at lower pressures when the LDA functional is used. The BLYP functional, by contrast, makes insulating structures relatively more stable than the metallic Cmca structure and predicts that the insulating Cmca-12 phase does not transform into the metallic Cmca phase until the pressure reaches $430 \mathrm{GPa}$. All three XC functionals suggest that the $\mathrm{P} 6_{3} / \mathrm{m}$ phase has the largest band gap and, consequently, the highest metallization pressure of the three insulating structures considered. The band gap of the Cmca-12 phase closes at the lowest pressure.

All three XC functionals also indicate that the insulating wide-band-gap hexagonal structure $\mathrm{P} 6_{3} / \mathrm{m}$ is stable in the low-pressure regime. Indeed, all three predict the same sequence of structural transitions, $\mathrm{P} 6_{3} / \mathrm{m} \rightarrow \mathrm{C} 2 / \mathrm{c}$ $\rightarrow$ Cmca-12 $\rightarrow$ Cmca, although at different pressures. They also agree that the band gap of the Cmca-12 structure (which is insulating at low pressure) closes before the transition to the Cmca structure (which is metallic at low pressure) takes place. However, the quantitative disagreements between results obtained using different XC functionals are so large that it is barely possible to compare the calculated DFT phase diagram with experiment. Assuming that the I $\rightarrow$ II and II $\rightarrow$ III phase transitions observed experimentally at around 110 and $150 \mathrm{GPa}$ are related to structural transformations, different functionals ascribe qualitatively different structural transitions to each. The PBE phase diagram predicts a transition from $\mathrm{P}_{3} / \mathrm{m}$ to $\mathrm{C} 2 / \mathrm{c}$ at around $105 \mathrm{GPa}$, which could be the $\mathrm{I} \rightarrow \mathrm{II}$ transition. The LDA and BLYP phase diagrams show no structural transformations near $110 \mathrm{GPa}$ but two different transformations, $\mathrm{P} 6_{3} / \mathrm{m} \rightarrow \mathrm{C} 2 / \mathrm{c}$ and $\mathrm{C} 2 / \mathrm{c} \rightarrow$ Cmca-12, in the pressure range 150-160 GPa, possibly related to the II $\rightarrow$ III transition. None of the three XC functionals is able to explain both phase transitions.

\section{DYNAMIC RESULTS AND DISCUSSION}

The treatment of nuclear quantum effects (ZPE) within DFT is a challenging problem, but is especially 
important in hydrogen due to the small nuclear mass. Indeed, we show below that including the proton ZPE has a large effect on the phase diagram. DFT is also used to study other properties that depend on lattice vibrations, including Raman and IR spectra and the electron-phonon interaction. 43 s in the static case, the question arises as to how these dynamical aspects of the behavior of highpressure solid hydrogen are affected by the choice of XC functional. Answering this question is more important in the dynamic than the static case, since the experimental techniques most widely used to study high-pressure solid hydrogen are Raman and IR spectroscopy, both of which are interpreted using phonon calculations $\underline{45}$

Figure 7 shows the phonon densities of states of the Cmca-12 and C2/c structures calculated using the LDA, $\mathrm{PBE}$ and BLYP XC functionals. The principal effect of the choice of functional is on the size of the phonon band gap, which depends on pressure and decreases as the pressure increases. As in the case of the electronic band gap, the BLYP and LDA functionals give the largest and smallest phonon gap, respectively. Surprisingly, at low frequencies $\left(<2200 \mathrm{~cm}^{-1}\right)$, the phonon spectra obtained using the three different $\mathrm{XC}$ functionals are quite similar. The XC functional has a much larger effect in the highfrequency regime $\left(>3500 \mathrm{~cm}^{-1}\right)$. As Fig. 7 shows, the LDA, PBE and BLYP functionals all predict two strong modes above the phonon gap, but at significantly different frequencies. The uncertainties in the frequencies of these modes complicate the comparison of computational and experimental Raman and IR spectra. To illustrate this difficulty, we have investigated the sensitivity of the calculated IR spectrum of $\mathrm{C} 2 / \mathrm{c}$, Cmca- 12 , and $\mathrm{P}_{3} / \mathrm{m}$ phases at $175 \mathrm{GPa}$ to the choice of $\mathrm{XC}$ functional.

As illustrated in Fig. 8 the computed IR spectrum of the $\mathrm{C} 2 / \mathrm{c}$ phase exhibits a single strong vibron peak, although the peak intensity depends on the choice of XC functional. The peak frequencies calculated using the LDA, PBE, and BLYP functionals are approximately 3800,4200 , and $4600 \mathrm{~cm}^{-1}$, respectively. In the case of the Cmca-12 phase, all three functionals produce two peaks of similar intensity in the high frequency regime. The LDA peak frequencies are 3500 and $3750 \mathrm{~cm}^{-1}$; the PBE frequencies are 3820 and $4050 \mathrm{~cm}^{-1}$; and the BLYP frequencies are 4250 and $4460 \mathrm{~cm}^{-1}$. The simulated IR spectrum of the $\mathrm{P} 6_{3} / \mathrm{m}$ phase shows a single highfrequency peak, which appears at 4250, 4470, or 4860 $\mathrm{cm}^{-1}$ for the LDA, PBE, and BLYP functionals, respectively.

Experimental IR spectra ${ }^{8}$ taken at $158 \mathrm{GPa}$ show a single strong vibron peak with a frequency of $4400 \mathrm{~cm}^{-1}$. As the pressure increases, the frequency of this peak decreases, reaching $4300 \mathrm{~cm}^{-1}$ at $208 \mathrm{GPa}$, suggesting a frequency close to $4350 \mathrm{~cm}^{-1}$ at $\mathrm{P}=175 \mathrm{GPa}$. Experiment also shows 8 that the vibron intensity below $150 \mathrm{GPa}$ is approximately three orders of magnitude lower than at higher pressures. Although no XC functional yields the experimental IR frequency accurately, all indicate that the $\mathrm{C} 2 / \mathrm{c}$ phase has a very strong IR peak in roughly the right frequency range. As pointed out by Pickard and Needs, 15 this observation is consistent with the suggestion that phase III has the $\mathrm{C} 2 / \mathrm{c}$ structure. Regardless of the choice of $\mathrm{XC}$ functional, the $\mathrm{C} 2 / \mathrm{c}$ vibron peak is much more intense than the vibron peaks for the other structures studied.

The effect of ZPE on the phase diagram is normally studied by applying a simple, additive, pressuredependent correction to the static ground-state total energy. The zero-point motion is assumed to be harmonic and the phonon frequencies calculated as a function of volume using DFT. This was the procedure adopted here. The limitations of the harmonic approximation can be overcome using DFT-based path-integral molecular dynamics, 47 but this is very costly and still relies on the accuracy of the XC functional, which is clearly in doubt. Ground-state QMC simulations treating both the electrons and protons as quantum mechanical particles ${ }^{48}$ do not rely on an approximate $\mathrm{XC}$ functional but are even more expensive. Furthermore, only a few QMC simulations of this type have ever been attempted and there are good reasons to doubt their accuracy in practice.

Figure 9 summarizes the results of our proton ZPE calculations using the LDA, PBE and BLYP functionals. The proton ZPEs of all the structures investigated, Cmca-12, C2/c, $\mathrm{P} 6_{3} / \mathrm{m}$, and Cmca, are shown in the pressure range of phases I, II, and III. It is clear that the ZPE increases with increasing pressure regardless of the crystal structure or choice of XC functional, and that the BLYP and LDA functionals give the largest and smallest ZPEs in all cases. For the insulating phases, Cmca-12, $\mathrm{C} 2 / \mathrm{c}$, and $\mathrm{P} 6_{3} / \mathrm{m}$, increasing the pressure also increases the differences between the proton ZPEs obtained using different $\mathrm{XC}$ functionals. For the metallic Cmca phase, these differences are approximately independent of pressure. The differences between the proton ZPEs of the candidate crystal structures can be comparable to the differences between their static total energies. Therefore, structure searching algorithms including an approximate ZPE should be considered.

The crystal structures investigated here were all mechanically stable and fully relaxed in the supercells used for structure searching, but the existence of small imaginary phonon frequencies indicates that some may be weakly unstable with respect to structural distortions on longer length scales $\stackrel{15}{1}$ The imaginary phonon frequencies are relatively small, however, and their number can be reduced by time-consuming small relaxations of the structures in larger supercells.

The static phase diagrams presented in Fig. 5 assume that the protons are infinitely massive. By adding the proton ZPE to the static results we obtain the dynamic phase diagrams reported in Fig. 10. The LDA dynamic phase diagram shows three phase transformations: $\mathrm{P} 63 / \mathrm{m}$ to $\mathrm{C} 2 / \mathrm{c}$ at around $50 \mathrm{GPa}, \mathrm{C} 2 / \mathrm{c}$ to Cmca12 at around $170 \mathrm{GPa}$, and the Cmca-12 to Cmca metalinsulator transition at around $200 \mathrm{GPa}$. The PBE and BLYP dynamic phase diagrams place the $\mathrm{P} 6_{3} / \mathrm{m}$ to $\mathrm{C} 2 / \mathrm{c}$ 
transition at $110-130 \mathrm{GPa}$. This suggests that the III phase transition observed experimentally at $110 \mathrm{GPa}$ corresponds to the $\mathrm{P} 6_{3} / \mathrm{m}$ to $\mathrm{C} 2 / \mathrm{c}$ structural transformation, even though the IR data provides suggests that $\mathrm{C} 2 / \mathrm{c}$ corresponds to phase III. The dynamic PBE and BLYP phase diagrams agree that the Cmca-12 structure is never stable in the pressure range associated experimentally with phase III and that the $\mathrm{C} 2 / \mathrm{c}$ structure transforms directly, or almost directly, into the metallic Cmca structure. The transition to Cmca-12 proposed by Pickard and Needs $\frac{15}{}$ has not been observed experimentally ${ }^{\underline{8}}$ and a recent metadynamics calculation $\underline{49}$ also suggests that, at finite temperature, phase III may transform to Cmca without passing through Cmca-12. If the Cmca-12 phase is ever stable, it is only within a very narrow window just below the pressure of the transition to the metallic Cmca structure.

The inclusion of the effects of proton ZPE substantially decreases the metallization pressure. Although the PBE and BLYP dynamic phase diagrams can both be interpreted as in agreement with experimental results concerning metallization, they do not agree with the same experimental results. Eremets and Troyan claim that ${ }^{7}$ hydrogen transforms into a metal at 260-270 GPa: the conductance increases sharply and changes little on increasing the pressure to $300 \mathrm{GPa}$ or cooling to $30 \mathrm{~K}$; and the sample reflects light well. As is shown in Fig. 10(b), the PBE dynamic phase diagram supports this claim: $\mathrm{C} 2 / \mathrm{c}$ remains the most stable structure throughout phases II and III and transforms directly or almost directly into the metallic Cmca phase at 240-250 GPa. On the other hand, Hemley and coworkers ${ }^{-8}$ see no evidence of a metallic state up to 360 GPa, but report electronic properties consistent with semi-metallic behaviour. The BLYP dynamic phase diagram, Fig. 10(c), shows a metal-insulator transition at 350-360 GPa, related to a phase transition from the $\mathrm{C} 2 / \mathrm{c}$ structure to the Cmca structure. The dynamic phase diagrams also suggest that metallization of high-pressure solid hydrogen happens through a molecular structural transformation, not band-gap closure. The pressure at which the band gap of the $\mathrm{C} 2 / \mathrm{c}$ phase closes, as shown in Table I and also as calculated using the more accurate $G W$ approximation, $\stackrel{29}{,}$ is considerably higher than the pressure at which the transition to the metallic Cmca structure takes place. Since metallization is likely to take place via a structural transformation, the failure of DFT to produce accurate band gaps is unlikely to be relevant.

Last, but not least, we ask why DFT is unable to reproduce the phase diagram of high-pressure solid hydrogen. We believe that there are two main problems. One is caused by the very small enthalpy differences of a few $\mathrm{meV}$ per proton between phases. Exhaustive tests for molecules, where accurate results can be obtained using quantum chemical methods, have shown that no current $\mathrm{XC}$ functional is consistently capable of achieving such accuracy $\stackrel{32}{=}$ The second is the many-body self-interaction (XC-SI) error present in the XC functionals used here.
A systematic investigation ${ }^{50}$ found the XC-SI errors of the LDA, GGA, and BLYP total energies of a single $\mathrm{H}_{2}$ molecule to be $1.264,-0.126$, and $0.0846 \mathrm{eV}$, respectively. These values are more than two orders of magnitude larger than the DFT enthalpy differences between the crystal structures of high-pressure solid hydrogen. The GGA functional has a lower X-SIE than the LDA functional, but the C-SIE, which is negative by definition, is larger than the X-SIE in this case. The XC-SIE of the BLYP functional is relatively small because the LYP functional by construction does not suffer from the CSIE. In previous work ${ }^{28}$ we showed that the use of hybrid XC functionals, which generally suffer from smaller XC-SI errors than pure density functionals,,$\frac{32}{=}$ changes the phase diagram considerably.

\section{CONCLUSION}

This paper reported DFT calculations using the LDA, PBE and BLYP XC functionals of various proposed structures of solid molecular hydrogen at low temperature and high pressure. Static results obtained using all three XC functionals are consistent with the following scenario: at the highest pressures considered here, the Cmca-12 structure, which is insulating at low pressure but becomes semi-metallic with increasing pressure, transforms into the Cmca structure, which is metallic even at low pressure. Since the Cmca-12 and Cmca structures have the same space group, differing only in the number of hydrogen molecules per unit cell, this seems reasonable. More generally, as the pressure is increased, the band gaps of the insulating structures of solid hydrogen become smaller. As the band gaps decrease, we find that structures with wider gaps become progressively less stable relative to structures with smaller gaps, and a series of transitions to structures with smaller gaps takes place. This process culminates in the transition to the metallic Cmca structure.

The above scenario changes significantly when the effects of proton ZPE are added to the static phase diagram. Our ZPE calculations were based on the harmonic approximation, which is standard but may be problematic. However, it is clear from our results that the effects of proton ZPE are essential and must be taken into account, even if only approximately. Surprisingly, but perhaps fortuitously, the agreement between the PBE and BLYP dynamic phase diagrams is much better than the agreement between the corresponding static phase diagrams. In particular, both dynamic phase diagrams suggest that the experimentally observed I-II phase transition corresponds to a structural transformation from $\mathrm{P}_{3} / \mathrm{m}$ to $\mathrm{C} 2 / \mathrm{c}$, that $\mathrm{C} 2 / \mathrm{c}$ is the most stable of the structures considered over the whole pressure range of phases II and III, and that metallization happens via a molecular structural transformation from $\mathrm{C} 2 / \mathrm{c}$ to Cmca without passing through Cmca-12. The main difference between the PBE and BLYP dynamic phase diagrams is 
the pressure of the $\mathrm{C} 2 / \mathrm{c}$ to Cmca transition, which the PBE functional places at 240-250 GPa and the BLYP functional places at 350-360 GPa. As discussed above, both results can be interpreted as being in agreement with recent experimental results, but different and conflicting ones. The low-frequency $\left(<2200 \mathrm{~cm}^{-1}\right)$ parts of the phonon spectra of all structures considered are surprisingly independent of the choice of $\mathrm{XC}$ functional, but this is not true of the high-frequency vibronic parts. In general, it appears that the BLYP functional produces stiffer phonons than the PBE functional and considerably stiffer phonons than the LDA functional.

This work focused on four specific crystal structures recently proposed on the basis of $\mathrm{PBE}$ calculations $15 \mathrm{Al}$ though the phase diagrams obtained using different XC functionals differed substantially in quantitative terms, especially at the static level, the four structures always appeared in the same order as the pressure was increased.
We think it likely, however, that if we had considered a wider range of structures, we would have seen qualitative as well as quantitative differences between the phase diagrams calculated using different $\mathrm{XC}$ functionals. DFT is probably not accurate enough to allow reliable comparisons of the stabilities of the insulating, semi-metallic, and metallic phases of solid molecular hydrogen. Indeed, since simple DFT calculations fail to describe van der Waals interactions and severely underestimate band gaps, it would be unreasonably optimistic to expect such comparisons to be reliable. We conclude that using DFT results as the basis of our understanding of high-pressure solid hydrogen is likely to lead to serious errors. To obtain reliable results, it is almost certainly necessary to go beyond DFT and take many-body effects properly into account ${ }^{29}$ In any case, it is clear that more work needs to be done, both theoretically and experimentally, to understand solid molecular hydrogen at low temperature.
1 E. Wigner and H. B. Huntington, J. Chem. Phys. 3, 764 (1935).

2 S. A. Bonev, E. Schwegler, T. Ogitsu, and G. Galli, Nature (London) 431, 669 (2004).

3 N. W. Ashcroft, Phys. Rev. Lett. 21, 1748 (1968), Phys. Rev. Lett. 92, 187002 (2004).

4 E. Babaev, A. Sudbo, and N. W. Ashcroft, Nature (London) 431, 666 (2004).

${ }^{5}$ C. F. Richardson and N. W. Ashcroft, Phys. Rev. Lett. 78, 118 (1997).

${ }^{6}$ A. Alavi, M. Parrinello, and D. Frenkel, Science 269, 1252 (1995).

7 M. I. Eremets and I. A. Troyan, Nature Mater. 10, 927 (2011).

8 C-S. Zha, Z. Liu, and R. J. Hemley, Phys. Rev. Lett. 108, $146402(2012)$.

9 J. Kohanoff, S. Scandolo, G. L. Chiarotti, and E. Tosatti, Phys. Rev. Lett. 78, 2783 (1997)

10 Y. Akahama, M. Nishimura, H. Kawamura, N. Hirao, Y. Ohishi, and K. Takemura, Phys. Rev. B 82, 060101(R) (2010).

11 R. J. Hemley, H-k. Mao, A. F. Goncharov, M. Hanfland, and V. Struzhkin, Phys. Rev. Lett. 76, 1667 (1996).

12 V. Natoli, R. M. Martin, and D. Ceperley, Phys. Rev. Lett. 70, 1952 (1993); 74, 1601 (1995).

13 P. Loubeyre, R. LeToullec, D. Hausermann, M. Hanfland, R. J. Hemley, H. K. Mao, and L. W. Finger, Nature 383, 702 (1996).

14 C. J. Pickard and R. J. Needs, J. Phys.: Condens. Matter 23, 053201 (2011).

15 C. J. Pickard, and R. J. Needs, Nature Physics 3, 473 (2007).

16 A. R. Oganov, A. O. Lyakhov, and M. Valle, Acc. Chem. Res. 44, 227 (2011).

17 A. R. Oganov and C. W. Glass, J. Chem. Phys. 124244704 (2006).

18 D. M. Ceperley and B. J. Alder, Phys. Rev. B 36, 2092 (1987).

19 H. Chacham and S. G. Louie, Phys. Rev. Lett. 66, 64 (1991).
20 E. Kaxiras, J. Broughton, and R. J. Hemley, Phys. Rev.Lett. 67, 1138 (1991).

21 J. Kohanoff, S. Scandolo, S. de Gironcoli, and E. Tosatti, Phys. Rev. Lett. 83, 4097 (1999).

${ }^{22}$ K. A. Johnson and N. W. Ashcroft, Nature (London) 403, 632 (2000).

23 M. Stadele and R. M. Martin, Phys. Rev. Lett. 84, 6070 (2000).

24 A. R Oganov, Modern Methods of Crystal Structure Prediction. Wiley-VCH, Berlin. ISBN: 978-3-527-40939-6 (2010).

25 A. Mujica, Angel Rubio, A. Munoz, and R. J. Needs, Rev. Mod. Phys. 75863 (2003).

26 M. Martinez-Canales, A. R. Oganov, A. Lyakhov,Y. Ma, and A. Bergara, Phys. Rev. Lett. 102, 087005 (2009).

27 Y. Ma, A. R. Oganov, Y. Xie, Z. Li, and J. Kotakoski, Phys. Rev. Lett. 102, 065501 (2009).

28 Sam Azadi and Thomas D.Kuhne, JETP Lett. 95, 509 (2012).

29 S. Azadi, W. M. C. Foulkes, and T. D. Kuhne (submitted to New J. Phys.).

30 M. J. Gillan, F. R. Manby, M. D. Towler, and D. Alfe, J. Chem. Phys. 136, 244105 (2012) (and references therein).

31 B. Santra, A. Michaelides, and M. Schefer, J. Chem. Phys. 131, 124509 (2009).

32 A. J. Cohen, P. Mori-Sánchez, and W. Yang, Chem. Rev. 112, 289, (2012).

33 J. P. Perdew and A. Zunger, Pys. Rev. B 23, 5048 (1981).

34 J. P. Perdew, K. Burke, M. Ernzerhof, Phys. Rev. Lett. 77, 3865 (1996).

35 C. Lee, W. Yang, R. G. Parr, Phys. Rev. B 37, 785 (1988).

36 M. A. Morales, J. M. McMahon, C. Pierleoni, and D. M. Ceperley, Phys. Rev. Lett. 110, 065702 (2013).

37 P. Giannozzi et al., J. Phys.: Condens. Matt. 21, 395502 (2009).

38 S. Azadi, C. Cavazzoni, and S. Sorella, Phys. Rev. B 82, 125112 (2010).

39 V. Labet, P. Gonzalez-Morelos, R. Hoffmann, and N. W. Ashcroft. J. Chem. Phys. 136074501 (2012).

${ }^{40}$ V. Labet, R. Hoffmann, and N. W. Ashcroft. J. Chem. Phys. 136 074502, 074503, 074504 (2012). 
41 A. Marini, C. Hogan, M. Gruning and D. Varsano, Comput. Phys. Commun. 180,1392 (2209).

42 J. M. McMahon, and D. M. Ceperley, Phys. Rev. Lett. 106, 165302 (2011).

43 J. M. McMahon, M. A. Morales, C. Pierleoni, and D. M. Ceperley, Rev. Mod. Phys. 84, 1607, (2012).

44 H. K. Mao, and R. J. Hemley, Rev. Mod. Phys. 66, 671 (1994).

45 S. Baroni, S. de Gironcoli, and A. Dal Corso, Rev. Mod. Phys. 73, 515 (2001).

46 I. Goncharenko, and P. Loubeyre, Nature (London) 435,
1206 (2005).

47 Xin-Zheng Li, Brent Walker, Matthew I. J. Probert, Chris J. Pickard, Richard J. Needs, and Angelos Michaelides, J. Phys.: Condens. Matter 25, 085402 (2013).

48 D. M. Ceperley, and B. J. Alder, Phys. Rev. B 36, 2092 (1987).

49 H. Liu, L. Zhu, W. Cui, and Y. Ma, J. Chem. Phys. 137, 074501 (2012).

50 V. Polo, E. Kraka, and D. Cremer, Molecular Physics 100, 1771 (2002). 

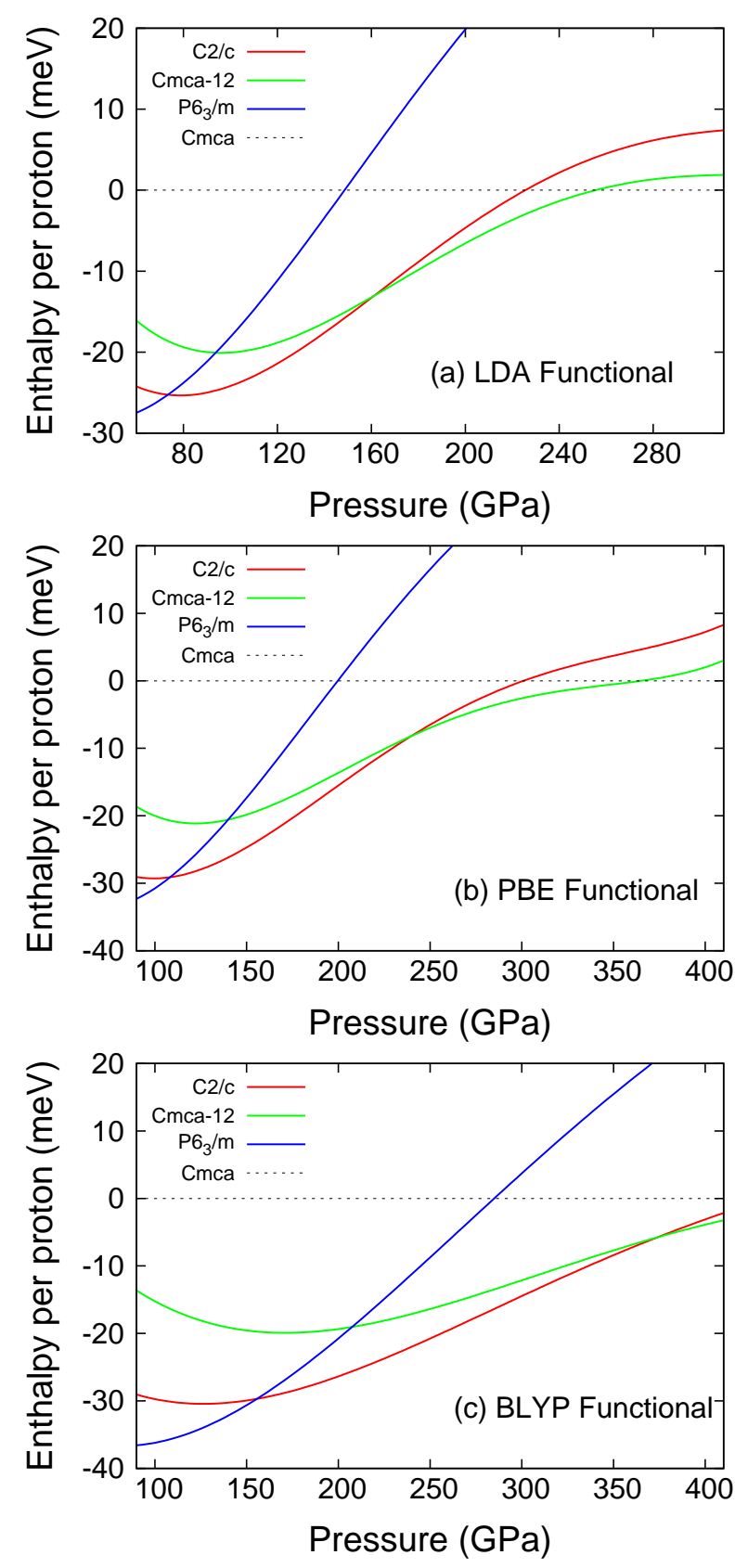

FIG. 5. (color online). Enthalpy per proton as a function of pressure calculated using three different XC functionals: (a) LDA, (b) PBE, (c) BLYP. The static lattice enthalpies of three different insulating crystal structures are reported relative to the enthalpy of the metallic Cmca structure. The Cmca-12 structure, which is insulating at low pressure, transforms into the metallic Cmca structure at 260, 370 or 430 GPa according to the LDA, PBE and BLYP approximations, respectively. 

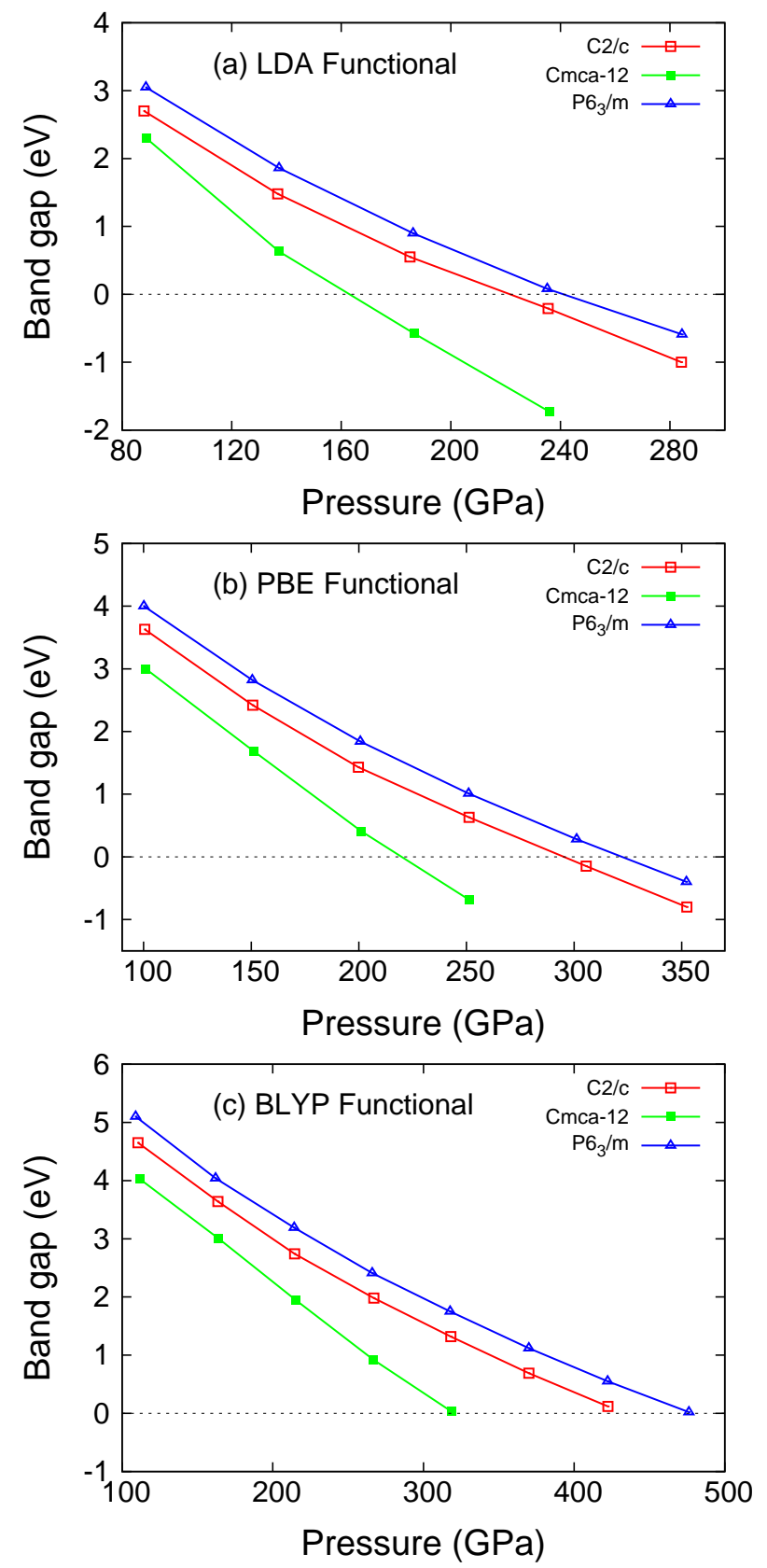

FIG. 6. (color online). Single-particle band gaps versus pressure calculated using (a) the LDA, (b) the PBE, and (c) the BLYP functionals. The BLYP band gap is larger than the LDA and PBE gaps and consequently yields larger metallization pressures. The $\mathrm{P} 6_{3} / \mathrm{m}$ and Cmca- 12 phases have the highest and lowest metallization pressures, respectively, regardless of the $\mathrm{XC}$ functional used. 

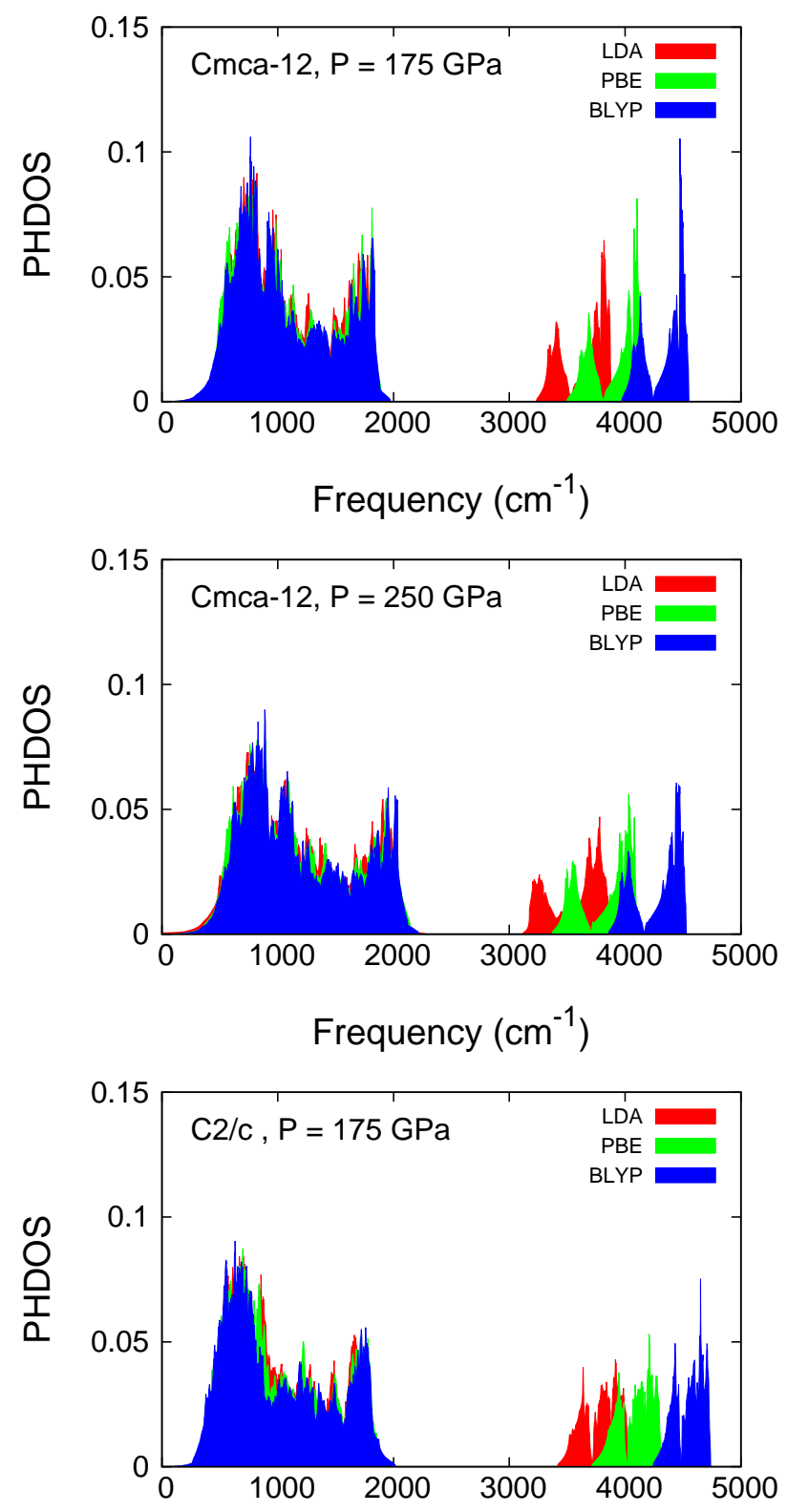

Frequency $\left(\mathrm{cm}^{-1}\right)$

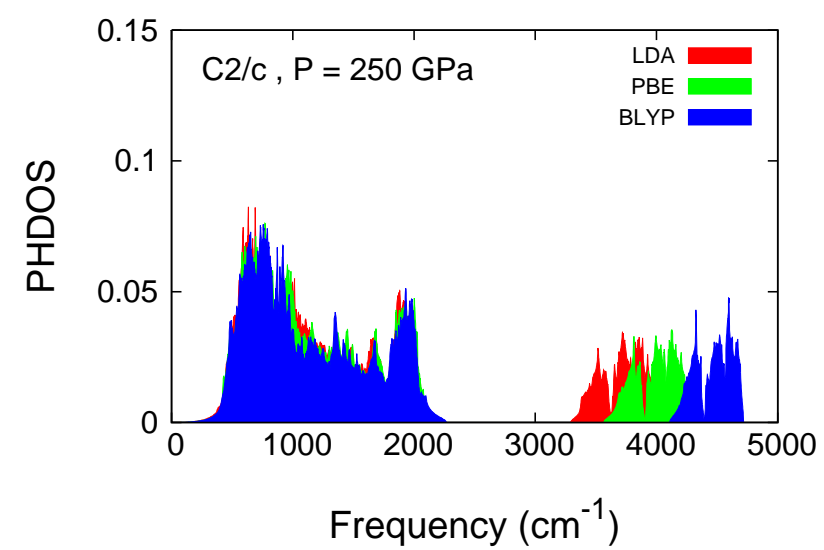

FIG. 7. (color online). The phonon densities of states of the Cmca- 12 and $\mathrm{C} 2 /$ c structures calculated using the LDA, PBE and BLYP functionals at 175 and $250 \mathrm{GPa}$. All three functionals yield almost the same low frequency $\left(<2200 \mathrm{~cm}^{-1}\right)$ phonon spectrum. The frequencies of the vibron modes in the high-frequency regime $\left(>3500 \mathrm{~cm}^{-1}\right)$ depend strongly on the choice of functional. 

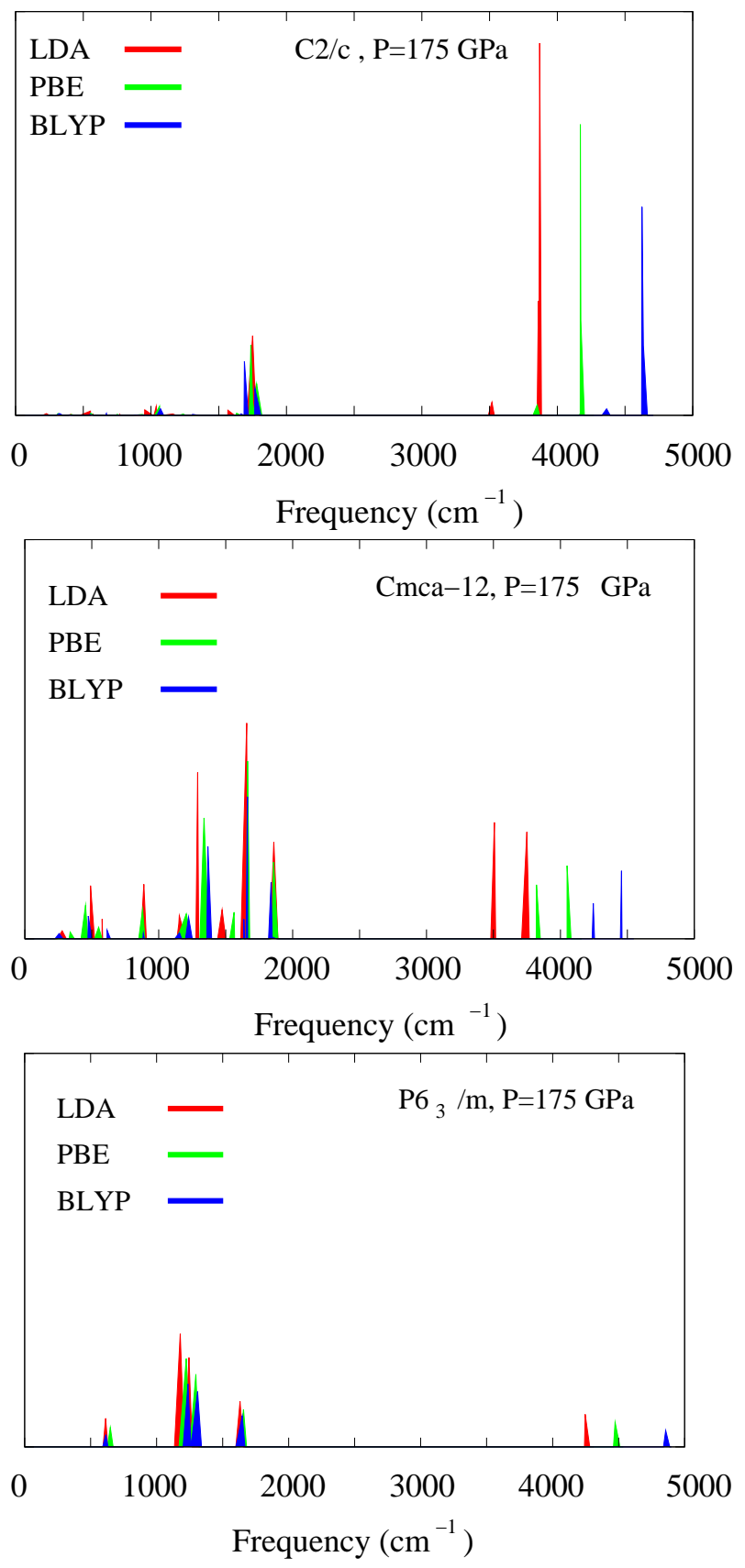

FIG. 8. (color online). Simulated IR spectra of the $\mathrm{C} 2 / \mathrm{c}$, Cmca- 12 and $\mathrm{P} 63 / \mathrm{m}$ phases at $175 \mathrm{GPa}$. The intensity scale used for the $\mathrm{P} 6_{3} / \mathrm{m}$ phase is double that used for the Cmca-12 and $\mathrm{P} 6_{3} / \mathrm{m}$ phases. 

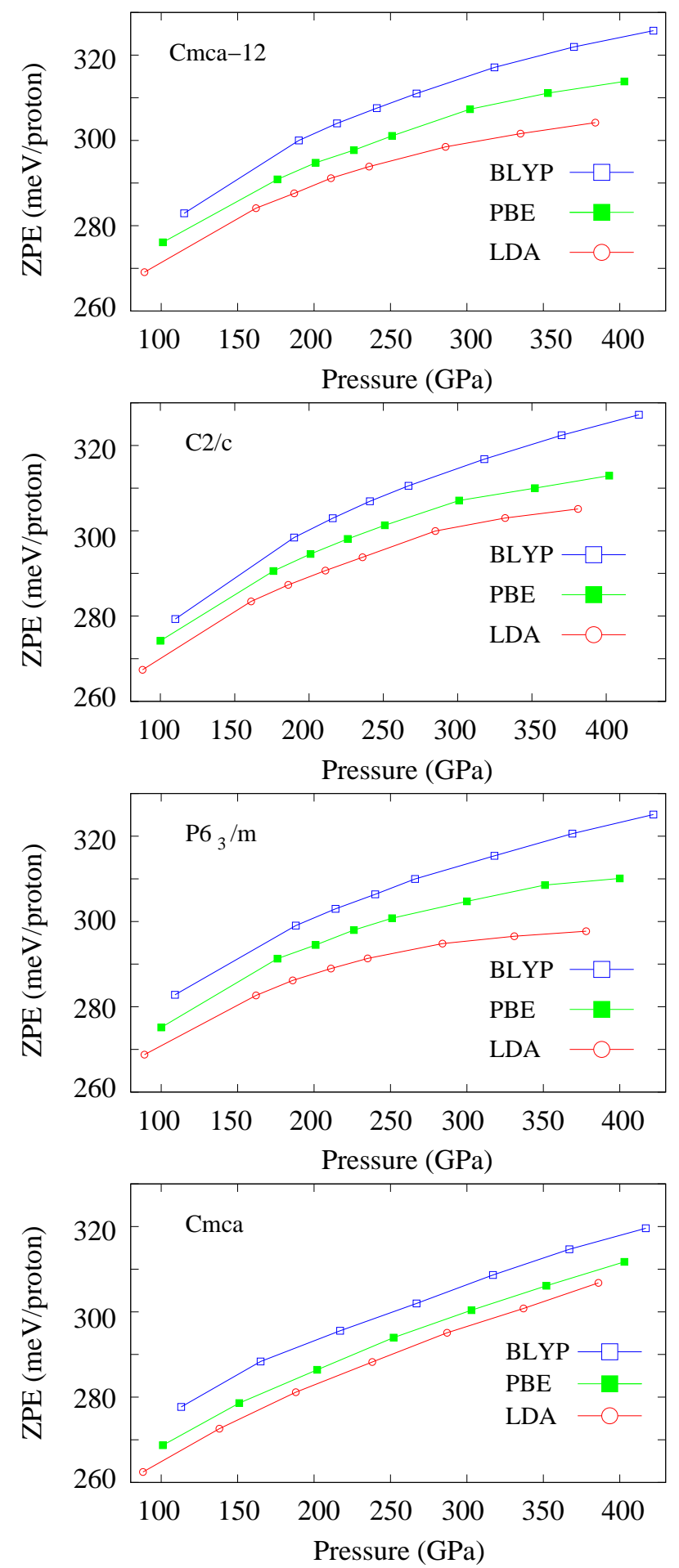

FIG. 9. (color online). The proton zero-point energies of the Cmca-12, C2/c, $\mathrm{P}_{3} / \mathrm{m}$ and Cmca crystal structures within the pressure range of phases I,II, and III as calculated using the LDA, PBE and BLYP functionals. The ZPE per proton increases with pressure. At any given pressure, the difference between ZPEs calculated using the BLYP and LDA functionals is more than $10 \mathrm{meV} /$ proton; this is large in comparison with the few $\mathrm{meV} /$ proton enthalpy differences between structures. 

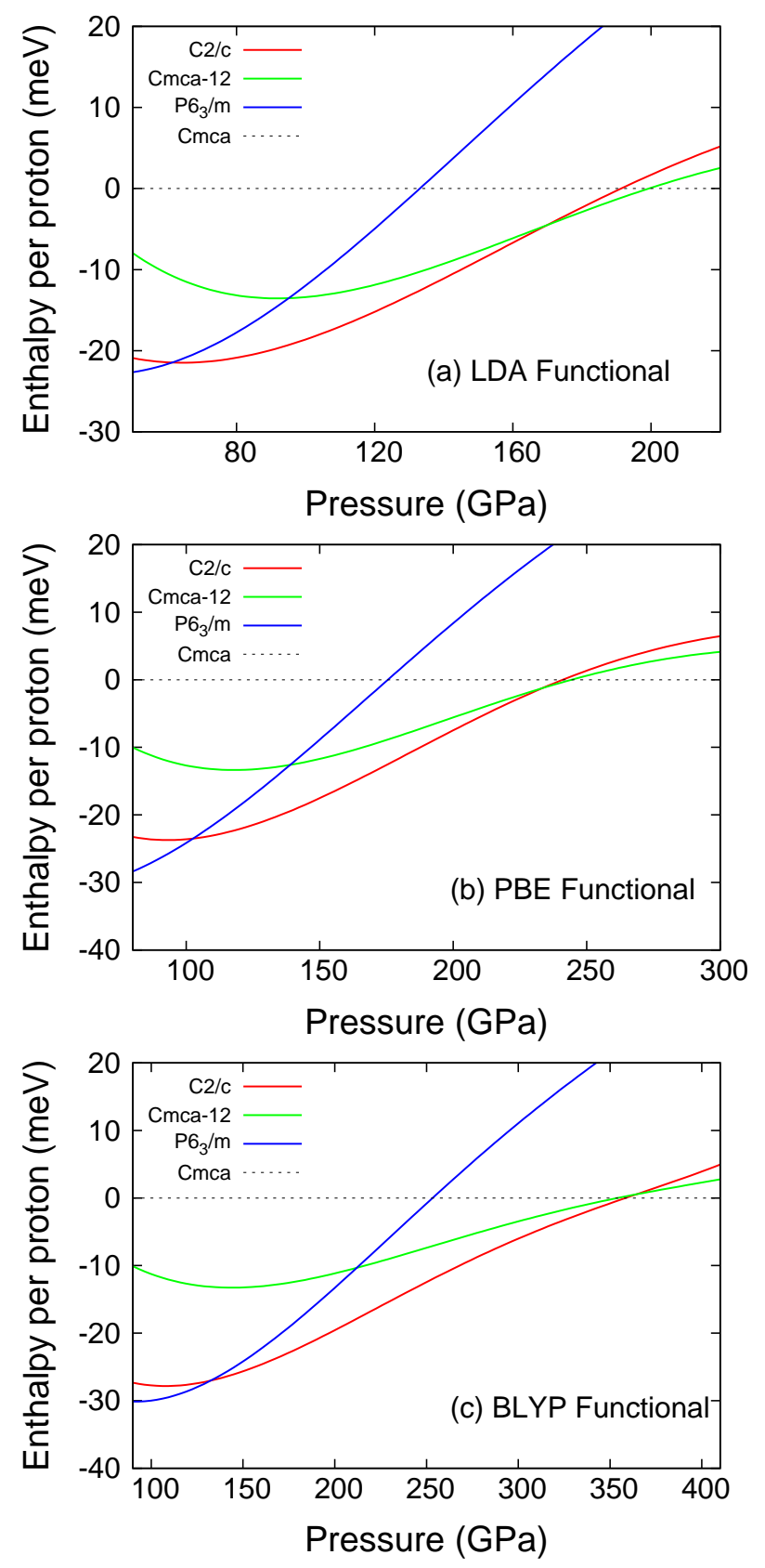

FIG. 10. (color online). Enthalpy per proton included ZPE (dynamic enthalpy) as a function of pressure calculated using three different XC functionals: (a) LDA, (b) PBE, (c) BLYP. The dynamic lattice enthalpies of three different insulating crystal structures are reported relative to the enthalpy of the metallic Cmca structure. 